\title{
Neutron Incident Angle and Energy Distribution at Vacuum Vessel for Beam-Injected Deuterium Plasmas in the Large Helical Device*)
}

\author{
Shota SUGIYAMA, Hideaki MATSUURA and Takuya GOTO ${ }^{1)}$ \\ Department of Applied Quantum Physics and Nuclear Engineering, \\ Kyushu University, 744 Motooka, Fukuoka 819-0395, Japan \\ 1) National Institute for Fusion Science, 322-6 Oroshi-cho, Toki 509-5292, Japan
}

(Received 30 November 2015 / Accepted 26 January 2016)

\begin{abstract}
Assuming a deuterium-beam-injected deuterium plasma confined in the Large Helical Device (LHD), the incident angle distribution and energy spectra of neutrons at various wall positions are evaluated by considering the complex shape of the vacuum vessel and the deuteron velocity distribution function. The effect of anisotropy of this function on the incident flux, the energy spectra, and the incident angle distribution of the neutrons to the vacuum vessel, are examined. In this paper, the neutron spectra are shown to depend on the wall position and the incident direction because of the formation of anisotropic high-energy tail in the deuteron velocity distribution function and the characteristic shape of the vacuum vessel of the LHD.
\end{abstract}

(C) 2016 The Japan Society of Plasma Science and Nuclear Fusion Research

Keywords: fast-ion velocity distribution function, neutron emission spectrum, beam-injected deuterium plasma, neutron incident angle distribution, neutron incident energy spectrum, Large Helical Device

DOI: $10.1585 /$ prr.11.2403049

\section{Introduction}

In toroidal devices, neutron wall loading and incident flux to the first wall depend on the poloidal angular wall position because of the shape of the torus wall [1,2]. The shape of the first wall may cause an additional nonuniformity of the neutron incident flux because the vacuum vessel of helical devices has a dumbell-shaped poloidal cross section. The neutron flux has been evaluated for neutron measurement, e.g., for in situ calibration of neutron monitor [3], use of Monte Carlo simulation in the Large Helical Device (LHD) by approximately considering the complex shape of the vacuum vessel. Neutron emission profiles can be considered for these calculations [4]. However, the neutron incident energy spectrum and the incident angle distribution to the first wall as a differential amount of the neutron flux have not been evaluated. In order to improve the efficiency of neutron measurements [5] and maintenance of diagnostics equipment, the neutron energy spectra for every incident angle at all wall positions should be grasped.

Energetic ions contribute to the formation of highenergy ion tails in ion velocity distribution functions $[6,7]$. As a result of this formation, the neutron emission spectrum is modified from a Gaussian distribution [6,7]. In neutral-beam-injection (NBI) heated plasmas, the highenergy ion tail and the non-Gaussian component in the neutron emission spectrum have anisotropic distributions because beam particles are injected in a particular direction. An effect of the anisotropy of emitted neutrons ap-

author'se-mail: s-sugi@nucl.kyushu-u.ac.jp

*) This article is based on the presentation at the 25th International Toki Conference (ITC25). pears when they collide with the first wall. Because incident neutrons produced by suprathermal reactions have an anisotropic spectrum, the incident directions of neutrons to the first wall are also anisotropic when the high-energy ion tail is formed. This trend could be seen in all toroidal devices. In addition to the geometrical effects of the first wall, anisotropic high-energy ion tail formation in the fuel ion velocity distribution function affects the neutron flux distribution, the energy spectra, and the incident angle distribution.

In this paper, assuming deuterium-beam-injected deuterium plasmas confined in the LHD, the incident angle distribution and energy spectra of neutrons at all wall positions are evaluated by considering the complex shape of the vacuum vessel and the deuteron velocity distribution function. The neutron spectra differ significantly depending on the wall position and the incident direction.

\section{Analysis Model}

We suppose that the position of the magnetic axis is $R_{\mathrm{ax}}=3.6 \mathrm{~m}$, which is shifted inward from the major radius of the device $R=3.9 \mathrm{~m}$. The bulk deuteron density $n_{\mathrm{d}}(\rho)=2 \times 10^{19} \mathrm{~m}^{-3}$, and the deuteron temperature $T_{\mathrm{d}}(\rho)=10\left(1-\rho^{2}\right) \mathrm{keV}$, where $\rho$ is the normalized radius, are assumed. The position of the magnetic axis corresponds to $\rho=0$. We assumed that the deuterium beam is tangentially injected at the magnetic axis, and that the high-energy ion tail in the deuteron velocity distribution function is formed in the beam-injected direction. An NBI power of $5 \mathrm{MW}$ and energy of $180 \mathrm{keV}$ are also assumed. We used the sum of the Maxwellian and the slowing-down 


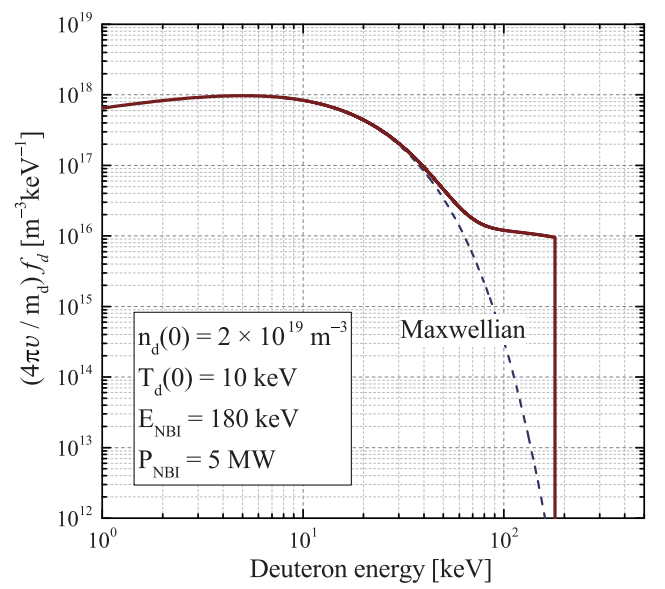

Fig. 1 The deuteron velocity distribution function on a magnetic axis. A beam energy of $180 \mathrm{keV}$, power of $5 \mathrm{MW}$, a bulk deuteron density $n_{\mathrm{d}}=2 \times 10^{19} \mathrm{~m}^{-3}$ and temperature $T_{\mathrm{d}}=$ $10 \mathrm{keV}$, are assumed.

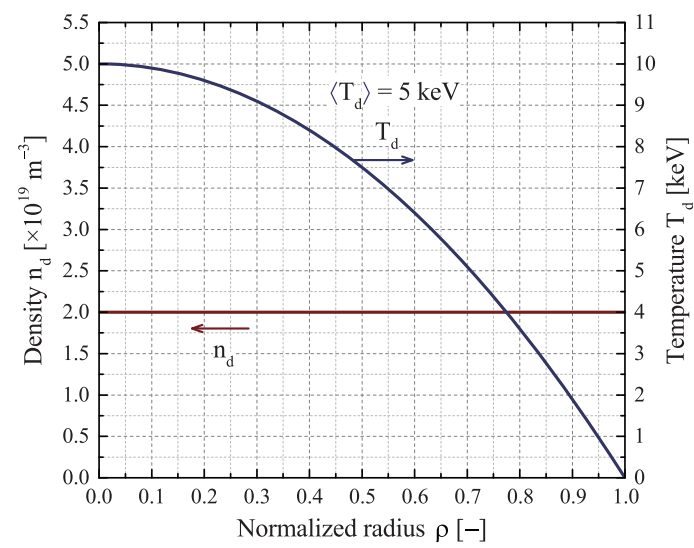

Fig. 2 Bulk deuteron temperature and density profiles. The averaged deuteron density $n_{\mathrm{d}}=2 \times 10^{19} \mathrm{~m}^{-3}$ and temperature $T_{\mathrm{d}}=5 \mathrm{keV}$, are assumed

distribution for the deuteron velocity distribution function. The deuteron velocity distribution function used in this calculation is shown in Fig. 1 while the assumed bulk deuteron profile is shown in Fig. 2.

The positions and directions of the energetic deuteron at the instant when the $\mathrm{D}(\mathrm{d}, \mathrm{n})^{3} \mathrm{He}$ reaction occurs are calculated by the orbit calculation with the LHD field. The cross section for the $\mathrm{D}(\mathrm{d}, \mathrm{n})^{3} \mathrm{He}$ reaction is taken from Bosch et al. [8] and Drosg et al. [9]. The orbit of a 180$\mathrm{keV}$ deuteron for 200 toroidal turns after generated at the magnetic axis with pitch angle $0^{\circ}$ is used as a test particle for this calculation.

The neutron emission direction and energy are calculated using the positions, directions, and energies of the energetic deuterons. The thermal motions of bulk deuterons are assumed to be isotropic. The neutron emission energy by the $\mathrm{D}(\mathrm{d}, \mathrm{n})^{3} \mathrm{He}$ reaction in the laboratory system is as follows [10]:

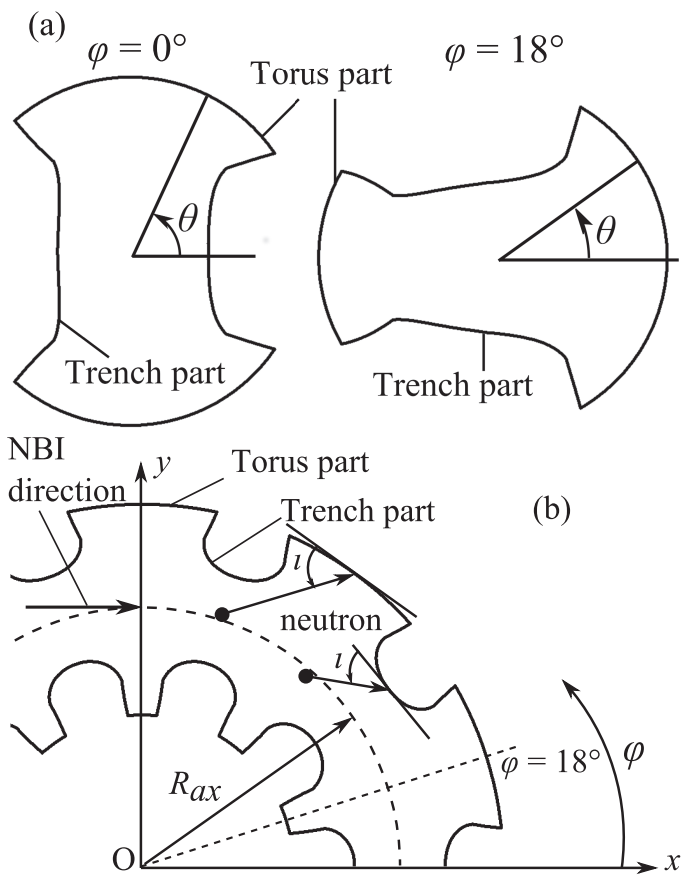

Fig. 3 The first wall shape (a) in the poloidal cross section and (b) in the horizontal cross section, and incident angle $\iota$.

$$
\begin{aligned}
E_{\mathrm{n}}= & \frac{1}{2} m_{\mathrm{n}} v_{0}^{2}+\frac{m_{3^{3} \mathrm{He}}}{m_{3 \mathrm{He}}+m_{\mathrm{n}}}\left(Q+E_{\mathrm{r}}\right) \\
& +v_{0} \cos \zeta \sqrt{\frac{2 m_{3^{3} \mathrm{He}} m_{\mathrm{n}}}{m_{3 \mathrm{He}}+m_{\mathrm{n}}}\left(Q+E_{\mathrm{r}}\right)},
\end{aligned}
$$

where $m_{\mathrm{n}\left({ }^{3} \mathrm{He}\right)}$ is the neutron $\left({ }^{3} \mathrm{He}\right)$ mass, $v_{0}$ is the centerof-mass velocity of the reacting deuterons, $\zeta$ is the angle between the center-of-mass velocity and the neutron velocity vectors in the center-of-mass system, $Q$ is the reaction $Q$-value, and $E_{\mathrm{r}}$ is the relative energy of the reacting deuterons.

The neutron incident points on the first wall and the neutron incident angles are calculated using the neutron emission direction and a mathematical expression of the first wall's shape. The incident angle $\iota$ of the neutron to the first wall is calculated as the angle between the neutron emission direction and the wall surface in the horizontal cross section. The first wall shape and the definition of the incident angle are shown in Fig. 3. In this paper, we define two parts, the trench part and the torus part in the poloidal cross section of the vacuum vessel of the LHD. The former is the part where helical coils are placed in, and the latter is the remaining part.

\section{Result and Discussion}

\subsection{Neutron emission spectrum}

The volume-averaged neutron emission spectrum by the $\mathrm{D}(\mathrm{d}, \mathrm{n})^{3} \mathrm{He}$ reaction is shown in Fig. 4 (a), and the double differential emission spectra are shown in Fig. 4(b), where $\chi$ is the angle between the neutron emission direction and toroidal axis. The neutron emission spectrum does 

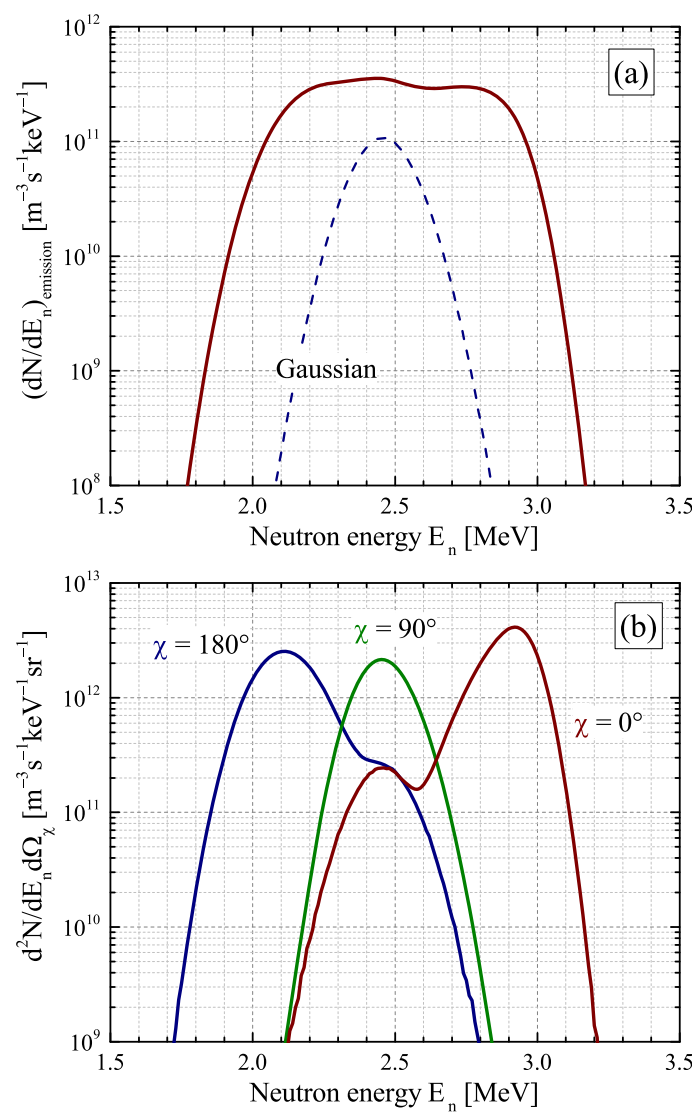

Fig. 4 (a) Volume-averaged and (b) double differential neutron emission spectra by the $\mathrm{D}(\mathrm{d}, \mathrm{n})^{3} \mathrm{He}$ reaction when the deuterium beam is tangentially injected in the deuterium plasma.

not obey the Gaussian distribution and the non-Gaussian component is formed with a range of energies from approximately 1.7 to $3.2 \mathrm{MeV}$. From Fig. 4 (b), the neutron that has the maximum energy can only be emitted in the $\chi=0^{\circ}$ direction, because the deuterium beam is tangentially injected, and the neutron emitted in the same direction as the center-of-mass velocity of the reacting deuterons has the maximum energy from Eq. (1). This anisotropy of the neutron emission affects the neutron incident spectra to the first wall.

\subsection{Neutron flux distribution}

The flux distribution of the non-Gaussian neutrons as the function of poloidal angle $\theta$ at the toroidal angle $\varphi=0^{\circ}$ is shown in Fig. 5. The flux in the trench part is much higher than that in the torus part. Because the trench part is closer to the plasma than the torus part, the neutrons can easily enter the trench part before they reach the torus part. The flux at $\theta=180^{\circ}$ is higher than that at $\theta=0^{\circ}$ because the vacuum vessel has a complex shape, and the distance between the vacuum vessel and the center of the plasma at $\theta=180^{\circ}$ is shorter than that at $\theta=0^{\circ}$. When we assume an isotropic plasma without beam injection in the magnetic configuration, the ratio of the flux at $\theta=180^{\circ}$ to that at

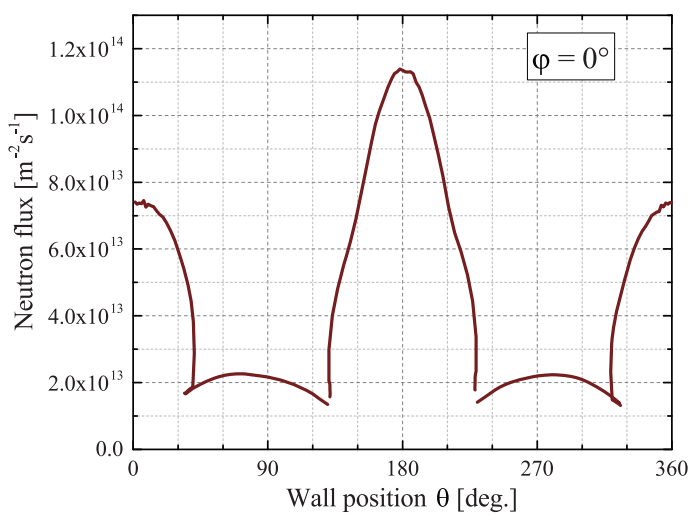

Fig. 5 Neutron flux distribution as a function of poloidal angle $\theta$ at $\varphi=0^{\circ}$.

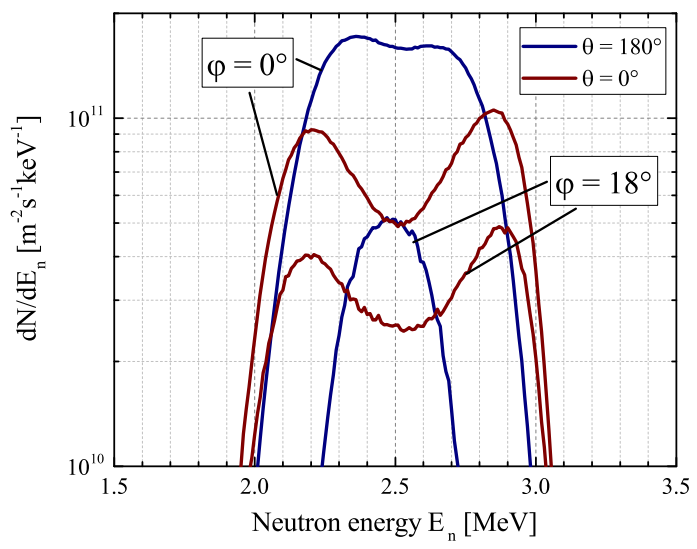

Fig. 6 Neutron incident energy spectra at $\varphi=0^{\circ}$ and $\theta=0^{\circ}$, $\varphi=0^{\circ}$ and $\theta=180^{\circ}, \varphi=18^{\circ}$ and $\theta=0^{\circ}$, and $\varphi=18^{\circ}$ and $\theta=180^{\circ}$.

$\theta=0^{\circ}$ becomes approximately $20 \%$ larger as compared with the beam-injected plasma. This is because neutrons emitted in the $\chi=0$ and $180^{\circ}$ directions, i.e., neutrons reaching the point $\theta=0^{\circ}$ on the vacuum vessel, increases because of anisotropic beam injection.

\subsection{Neutron incident energy spectra to the first wall}

The incident energy spectra of the non-Gaussian neutrons at $\varphi=0^{\circ}$ and $\theta=0^{\circ}, \varphi=0^{\circ}$ and $\theta=180^{\circ}, \varphi=18^{\circ}$ and $\theta=0^{\circ}$, and $\varphi=18^{\circ}$ and $\theta=180^{\circ}$ are shown in Fig. 6 . The neutron incident spectra depend on the wall position. Similar to the flux distribution, the spectra at $\varphi=0^{\circ}$ in the trench parts are larger than those at $\varphi=18^{\circ}$ in the torus parts. At $\theta=180^{\circ}$, neutrons with the maximum and minimum energy are not observed. In this calculation, neutrons emitted in the same direction as the NBI have maximum energy, whereas those emitted in the opposite direction have minimum energy. These neutrons can only enter at wall position $\theta=0^{\circ}$ from the geometric relationship between the neutron emission vector and the torus shape of the first wall. 

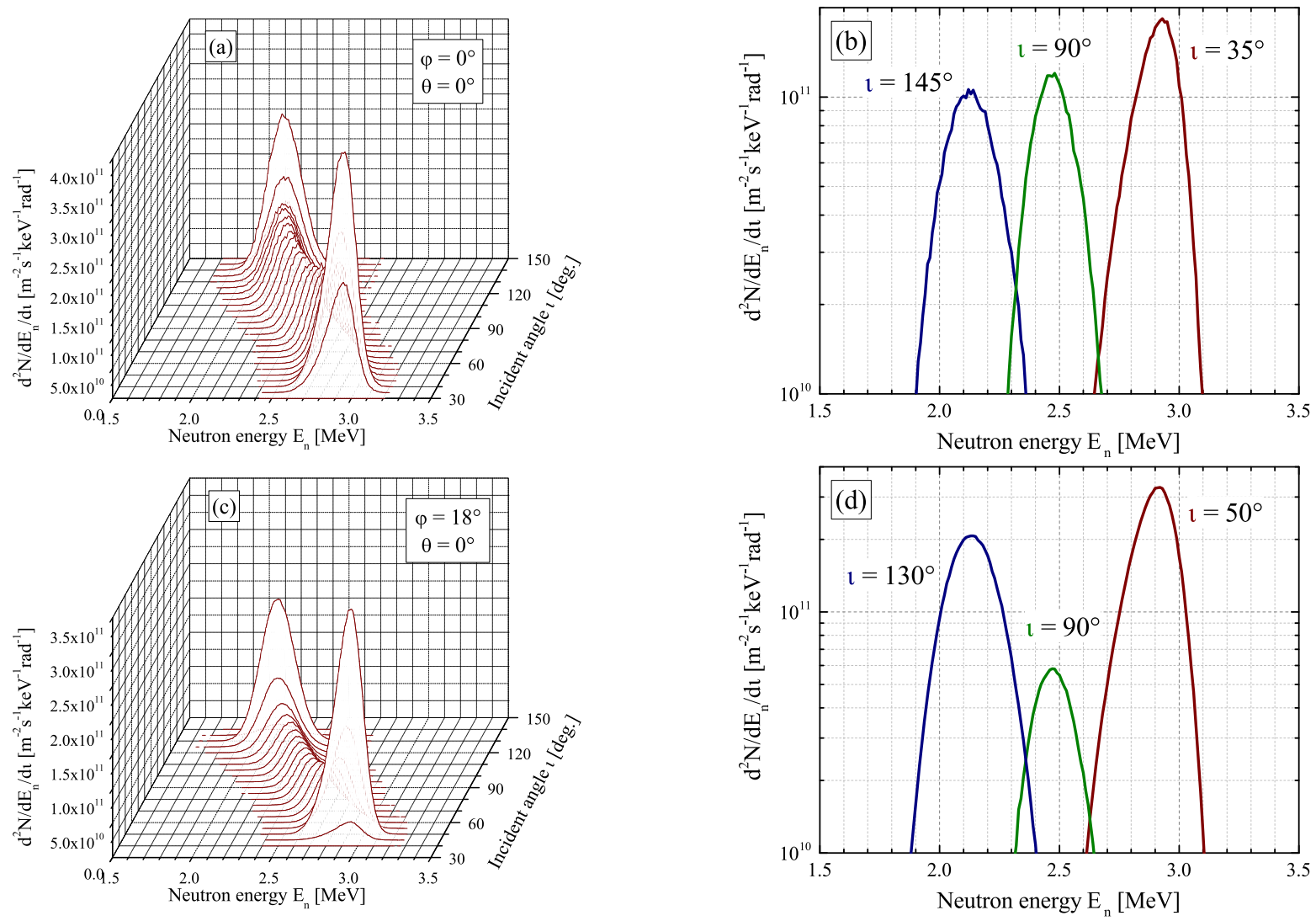

Fig. 7 Double differential neutron incident energy spectra at $\theta=0^{\circ}$ and $\varphi=0^{\circ}$ for (a) all incident angles, (b) $\iota=35,90$, and $145^{\circ}$, and at $\varphi=18^{\circ}$ for (c) all incident angles, and (d) $\iota=50,90$, and $130^{\circ}$.

\subsection{Neutron energy spectra for each incident angle to the first wall}

The incident angle and energy spectra of the nonGaussian neutrons at $\theta=0^{\circ}$ and (a) $\varphi=0^{\circ}$ at all incident angles, (b) $\varphi=0^{\circ}$ at $\iota=35,90$, and $145^{\circ}$, (c) $\varphi=18^{\circ}$ at all incident angles, and (d) $\varphi=18^{\circ}$ at $\iota=50,90$, and $130^{\circ}$ are shown in Fig. 7. The neutron incident angle correlates closely with the neutron energy. The neutron incident angle is determined by the geometric relationship between the shape of the first wall and the neutron emission direction. The neutrons emitted in the direction $\chi=0^{\circ}$ enter the first wall at an incident angle $\iota=35^{\circ}$ at wall position $\varphi=0^{\circ}$. Similarly, the neutrons emitted in the direction $\chi=180^{\circ}$ enter the first wall at an incident angle $\iota=145^{\circ}$ at the wall position $\varphi=0^{\circ}$.

The neutron spectrum at $\varphi=18^{\circ}$ and $\iota=50^{\circ}$ is approximately 1.5 times larger than that at $\varphi=0^{\circ}$ and $\iota=35^{\circ}$. However, at $\iota=90^{\circ}$ the spectrum at $\varphi=18^{\circ}$ is smaller than that at $\varphi=0^{\circ}$. This dependence of toroidal angle at the same poloidal angular position is not observed in tokamak devices.

As shown in Figs. 6 and 7, the neutron incident spectra depend on the wall position and the incident angle because of anisotropic neutron emission and the complex shape of the vacuum vessel of the LHD. This dependence is not observed in isotropic plasmas and uniform toroidal devices without trenches for helical coils. For accurate analyses of the spectra of non-Gaussian neutrons in the LHD, the anisotropic fuel ion velocity distribution function, orbit of energetic ion, and the complex shape of the vacuum vessel should be considered.

\section{Conclusion}

Assuming a deuterium-beam-injected deuterium plasma confined in the LHD, the neutron incident angle and energy spectra are examined by considering the complex shape of the vacuum vessel and the deuteron velocity distribution function. We presented the dependence of the neutron incident spectra on the wall position and the incident angle because of the anisotropic deuteron velocity distribution function and the shape of the vacuum vessel. The neutron spectrum at $\varphi=18^{\circ}$ and $\iota=50^{\circ}$ is approximately 1.5 times larger than that at $\varphi=0^{\circ}$ and $\iota=35^{\circ}$ at the same poloidal angular position $\theta=0^{\circ}$. This dependence of toroidal angle on the neutron incident spectra is a distinct feature of the helical device. Our results are important for improving the efficiency of neutron measurements and the maintenance of diagnostics equipment.

In this paper, we assumed that the high-energy ion tail is formed only in the NBI direction, and calculated the deuteron orbit without collisions. A more detailed calcu- 
lation with modifications to this analysis model (e.g., by considering the spatial and pitch-angle distribution of energetic ions) is the subject of a future study.

\section{Acknowledgement}

The authors are grateful to Prof. T. Watanabe for providing data of orbit calculation, fruitful discussion and his encouragement.

[1] P.P.H. Wilson et al., Fusion Eng. Des. 83, 824 (2008).

[2] J.C. Rivas et al., Fusion Sci. Technol. 64, 687 (2013).

[3] Y. Nakano et al., Rev. Sci. Instrum. 85, 11E116 (2014).
[4] M. Homma et al., Plasma Fusion Res. 10, 3403050 (2015).

[5] S. Sugiyama et al., Plasma Fusion Res. 10, 3403055 (2015).

[6] H. Matsuura and Y. Nakao, Phys. Plasmas 16, 042507 (2009).

[7] H. Matsuura et al., Plasma Phys. Control. Fusion 53, 035023 (2011).

[8] H.-S. Bosch and G.M. Hale, Nucl. Fusion 32, 611 (1992).

[9] M. Drosg and O. Schwerer, Production of monoenergetic neutrons between 0.1 and $23 \mathrm{MeV}$ : neutron energies and cross-sections, Handbook of Nuclear Activation Data (Vienna: IAEA) STI/DOC/10/273, ISBN 92-0-135087-2 (1987).

[10] H. Brysk, Plasma Phys. 15, 611 (1973). 